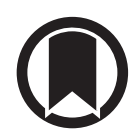

CrossMark

\title{
Effect of domiciliary oxygen therapy on exercise capacity and quality of life in patients with pulmonary arterial or chronic thromboembolic pulmonary hypertension: a randomised, placebo-controlled trial
}

\author{
Silvia Ulrich (10 ${ }^{1,3}$, Stéphanie Saxer ${ }^{1,2,3}$, Elisabeth D. Hasler ${ }^{1}$, Esther I. Schwarz ${ }^{1}$, \\ Simon R. Schneider ${ }^{1,2}$, Michael Furian ${ }^{1}$, Patrick R. Bader ${ }^{1}$, Mona Lichtblau (1) ${ }^{1}$ \\ and Konrad E. Bloch ${ }^{1}$
}

Affiliations: ${ }^{1}$ University Hospital Zurich, Dept of Respiratory Medicine, Pulmonary Hypertension Unit, Zurich, Switzerland. ${ }^{2}$ Dept of Health Sciences and Health Policy, University of Lucerne, Lucerne, Switzerland. ${ }^{3}$ Both authors contributed equally.

Correspondence: Silvia Ulrich, University Hospital Zurich, Dept of Respiratory Medicine, Pulmonary Hypertension Unit, Rämistrasse 100, CH-8091 Zürich, Switzerland. E-mail: silvia.ulrichQusz.ch

@ERSpublications

Domiciliary oxygen therapy improves 6-min walk distance and quality of life in patients with precapillary pulmonary hypertension who desaturate during exercise, and should thus be considered as an adjunct to medical therapy http://bit.ly/2M8H4iq

Cite this article as: Ulrich S, Saxer S, Hasler ED, et al. Effect of domiciliary oxygen therapy on exercise capacity and quality of life in patients with pulmonary arterial or chronic thromboembolic pulmonary hypertension: a randomised, placebo-controlled trial. Eur Respir J 2019; 54: 1900276 [https://doi.org/ $10.1183 / 13993003.002762019]$.

\section{ABSTRACT}

Study question: We investigated whether domiciliary oxygen therapy (DOXT) increases exercise capacity and quality of life in patients with pulmonary arterial or distal chronic thromboembolic pulmonary hypertension $(\mathrm{PAH} / \mathrm{CTEPH})$ presenting with mild resting hypoxaemia and exercise-induced oxygen desaturation.

Materials and methods: 30 patients with $\mathrm{PAH} / \mathrm{CTEPH}$, mean $\pm \mathrm{SD}$ age $60 \pm 15$ years, pulmonary artery pressure $39 \pm 11 \mathrm{mmHg}$, resting arterial oxygen saturation measured by pulse oximetry $\left(\mathrm{SpO}_{2}\right) \geqslant 90 \%, \mathrm{SpO}_{2}$ drop during a 6 -min walk test $\geqslant 4 \%$, on pulmonary hypertension-targeted medication, were randomised in a double-blind crossover protocol to DOXT and placebo (ambient air) treatment, each over 5 weeks, at $3 \mathrm{~L} \cdot \mathrm{min}^{-1}$ via nasal cannula overnight and when resting during the day. Treatment periods were separated by 2 weeks of washout. Co-primary outcomes were changes in 6 -min walk distance $(6 \mathrm{MWD}$, breathing ambient air) and physical functioning scale of the 36-item short-form medical outcome questionnaire during treatment periods.

Results: DOXT increased the 6MWD from baseline $478 \pm 113 \mathrm{~m}$ by a mean (95\% CI) of 19 (6-32) $\mathrm{m}$, and physical functioning from $52 \pm 29$ by $4(0-8)$ points. Corresponding changes with placebo were $1(-11-13) \mathrm{m}$ in $6 \mathrm{MWD}$ and $-2(-6-2)$ points in physical functioning. Between-treatment differences in changes were 6MWD $18(1-35) \mathrm{m}(\mathrm{p}=0.042)$ and physical functioning $6(1-11)$ points $(\mathrm{p}=0.029)$. DOXT significantly improved the New York Heart Association functional class versus placebo.

Answer to the question: This first randomised trial in PAH/CTEPH patients with exercise-induced hypoxaemia demonstrates that DOXT improves exercise capacity, quality of life and functional class. The results support large long-term randomised trials of DOXT in PAH/CTEPH. 


\section{Introduction}

In the absence of relevant lung disease, the major forms of precapillary pulmonary hypertension $(\mathrm{PH})$ are pulmonary arterial hypertension (PAH) and chronic thromboembolic $\mathrm{PH}(\mathrm{CTEPH})$. The main symptoms of PAH/CTEPH are exertional dyspnoea, impaired exercise performance and reduced quality of life [1-3]. Several pathophysiological mechanisms may account for the symptoms of PH. Typically, there is an excessive ventilatory drive leading to inefficient ventilation with high ventilatory equivalents for oxygen uptake and carbon dioxide output [4]. Impairments in cardiac output, ventilation-perfusion mismatch and right-left shunt along with a reduced pulmonary diffusing capacity result in arterial and mixed venous hypoxaemia that worsens $\mathrm{PH}$ even further by pulmonary vasoconstriction [5]. The already elevated ventilatory drive is additionally stimulated by progressive arterial hypoxaemia during exercise $[4,6]$. As a consequence, oxygen delivery to the muscles, the brain and other organs is reduced and exercise capacity is limited $[4,7,8]$.

We have shown that breathing oxygen-enriched air during cycle ergometry significantly increases maximal exercise capacity and almost doubles submaximal endurance time in patients with PAH/CTEPH and exercise-induced hypoxaemia [4]. This was related to a higher arterial oxygen content promoting oxygen availability in the brain and muscle tissue and reducing the excessive ventilatory response to exercise, thus enhancing ventilatory efficiency [4]. In a further randomised placebo-controlled double-blind trial in patients with PAH/CTEPH with nocturnal hypoxaemia and sleep-related breathing disturbances we found that nocturnal oxygen therapy over the course of 1 week improved the 6-min walk distance (6MWD) compared to placebo (ambient air) [9].

The treatment of precapillary $\mathrm{PH}$ includes targeted medication, supportive measures such as diuretics and exercise training, pulmonary endarterectomy or balloon-angioplasty in selected patients with CTEPH [10] and lung transplantation [1]. However, according to current guidelines, the role of supplemental oxygen therapy is not clearly established. Recommendations are based on studies in patients with chronic obstructive pulmonary disease (COPD) [11] and expert opinion suggesting that long-term oxygen therapy should be prescribed in patients with $\mathrm{PH}$ if the arterial oxygen tension $\left(\mathrm{PaO}_{2}\right)$ at rest is $<8 \mathrm{kPa}$ or if there is exercise-induced hypoxaemia with amelioration of symptoms by oxygen therapy during exercise [1]. In a systematic review of the literature we have identified only the two aforementioned randomised controlled trials [4, 9] evaluating the efficacy of oxygen therapy in PAH/CTEPH [12]. Therefore, the current randomised placebo-controlled trial tested the hypothesis that domiciliary oxygen therapy (DOXT) used for as many hours per day as possible over the course of 5 weeks improves the exercise performance and quality of life in patients with PAH/CTEPH who have exercise-induced hypoxaemia.

\section{Materials and methods \\ Study subjects}

Patients with PAH/CTEPH diagnosed according to current guidelines [1], aged 18-85 years, of both sexes were recruited among outpatients of the Pulmonary Hypertension Clinic, University Hospital Zurich. Study participants had to be in stable condition on therapy for $>4$ weeks. Patients with CTEPH had distal disease not suitable for endarterectomy or persistent $\mathrm{PH}$ after endarterectomy. Participants had to have a resting arterial oxygen saturation measured by pulse oximetry $\left(\mathrm{SpO}_{2}\right) \geqslant 90 \%$ and exercise-induced hypoxaemia, i.e. a drop in $\mathrm{SpO}_{2}$ by $\geqslant 4 \%$ to $\leqslant 92 \%$ during a 6 -min walk test (6MWT) breathing ambient air. Patients with severe hypoxaemia $\left(\mathrm{SpO}_{2}<90 \%\right.$ or $\mathrm{PaO}_{2}<7.3 \mathrm{kPa}$ at rest that would require long-term oxygen therapy according to current standards), with other forms of $\mathrm{PH}$, unstable condition, inability to follow study procedures, relevant comorbidities, obstructive sleep apnoea syndrome or pregnancy were excluded. The study lasted from January 2014 to January 2017. Participants provided written informed consent. The protocol was approved by the Cantonal Ethics Committee Zurich (2012-0538) and registered at www.clinicaltrials.gov (NCT01884012).

\section{Study design}

This randomised placebo-controlled double-blind crossover trial in patients with PAH or distal CTEPH receiving advanced $\mathrm{PH}$-targeted therapy evaluated the effect of DOXT on exercise performance and quality of life at the patient's home over the course of 5 weeks.

This article has supplementary material available from erj.ersjournals.com

Received: Feb 082019 | Accepted after revision: April 272019

This study is registered at clinicaltrials.gov (NCT01884012). Individual de-identified patient data will be shared with non-commercial entities upon a personalised request to the author. 
Minimal important differences $( \pm \mathrm{SD})$ for the co-primary outcomes were assumed as $35 \pm 50 \mathrm{~m}$ for the $6 \mathrm{MWD}$ [9] and $10 \pm 10$ points for the 36 -item short-form medical outcome questionnaire (SF-36) physical functioning scale [13]. To achieve a power of $80 \%, \alpha=0.05$, a minimal number of 26 participants was required. Accounting for possible dropouts, the goal was to include 30 participants.

Participants were randomised to a treatment sequence in balanced blocks of four using a computergenerated list. The study staff and participants were unaware of the type of administered treatment (double-blinded design). Unblinding was performed only after completion of data analysis.

\section{Interventions}

Patients received DOXT via an oxygen concentrator (EverFlow; Respironics, Zofingen, Switzerland) or ambient air (termed placebo in the following) via a modified device identical in appearance at a rate of $3 \mathrm{~L} \cdot \mathrm{min}^{-1}$ via a nasal cannula overnight and via a long tube for oxygen delivery at home during the day. The flow rate of $3 \mathrm{~L} \cdot \mathrm{min}^{-1}$ was selected because it was effective in our previous trial [9] and in order to achieve the highest possible effect and compliance while avoiding nasal discomfort and mucosal dryness. Patients were instructed to use each treatment for as long as possible, but for $\geqslant 16 \mathrm{~h} \cdot$ day $^{-1}$ over treatment periods. At the end of the first treatment period, a study nurse blinded to the type of treatment collected the first concentrator and, after a 2-week washout period, delivered the second concentrator for the subsequent 5 -week period. Operating hours of concentrators were recorded by a built-in counter.

\section{Assessments}

Medical history, physical examination and New York Heart Association (NYHA) functional class were evaluated. A 6MWT was performed while participants were breathing ambient air [14]. Quality of life was assessed using the SF-36 1-week recall form and the Cambridge Pulmonary Hypertension Outcome Review (CAMPHOR) [3, 15]. Arterial blood gas analysis of a radial artery blood samples (ABL90 FLEX blood gas analyser; Radiometer, Switzerland) and lung function tests were performed.

Echocardiography (Philips iE33; Philips, Zofingen, Switzerland) was performed at the end of the 5-week treatment periods to measure the right atrial and right ventricular areas, right ventricular fractional area change (RVFAC) and tricuspid annular plain systolic excursion (TAPSE) [16]. The maximal systolic velocity of the tricuspid regurgitation jet determined by Doppler ultrasound was used to determine the tricuspid pressure gradient (TPG). Right atrial pressure was estimated from the dimension and respiratory variability of the inferior cava vein.

Arterial blood gas analyses were obtained while patients were at rest and breathing ambient air (ABL blood gas analyser).

Respiratory sleep studies including pulse oximetry and nasal pressure swings (ApneaLink; ResMed, Basel, Switzerland) were performed in the last night of each 5-week treatment period at the patient's home. Mean nocturnal $\mathrm{SpO}_{2}$, oxygen desaturation index (ODI: number of $\mathrm{SpO}_{2}$ dips $\geqslant 4 \%$ lasting $\geqslant 10 \mathrm{~s} \cdot \mathrm{h}^{-1}$ ), pulse rate and apnoea-hypopnoea index (AHI: number of apnoeas + hypopnoeas per hour with reduction of breathing amplitude $<50 \%$ of baseline lasting $\geqslant 10 \mathrm{~s}$ and associated with a $\mathrm{SpO}_{2}$ dip $\geqslant 4 \%$ ) were determined [17]. Cognitive performance was tested using the Stroop test [18].

Over the course of the last week of each treatment period, physical activity was recorded using an accelerometer worn at the upper nondominant arm (SenseWear; BodyMedia, Pittsburgh, PA, USA) [19].

\section{Outcomes}

Co-primary outcomes were the changes in the 6MWD and in the SF-36 physical functioning scale from the beginning to the end of the 5-week treatment periods with oxygen and placebo. Secondary outcomes assessed at the end of each 5-week treatment period included NYHA functional class, results of questionnaire evaluations, echocardiography, sleep studies and actimetry. All outcomes measured during daytime were assessed while patients were breathing ambient air.

\section{Analysis}

Data are summarised as mean $\pm \mathrm{SD}$ and $\mathrm{n}(\%)$. Analysis of co-primary outcomes was performed in the intention-to-treat population with missing values replaced by multiple imputations using chained equations [20]. Treatment effects were assessed by computing linear mixed effects regression models with fixed effects of treatment (oxygen, placebo) and random effects of patients. This provided unadjusted differences of variables with $95 \%$ confidence intervals between oxygen and placebo treatment periods; adjusted treatment effects were computed by including treatment, age, sex and treatment order as independent variables into the models. Effect sizes were computed as mean change in a variable divided by the standard deviation of baseline; values of 0.2 were considered small and 0.5 moderate [21]. Analyses 


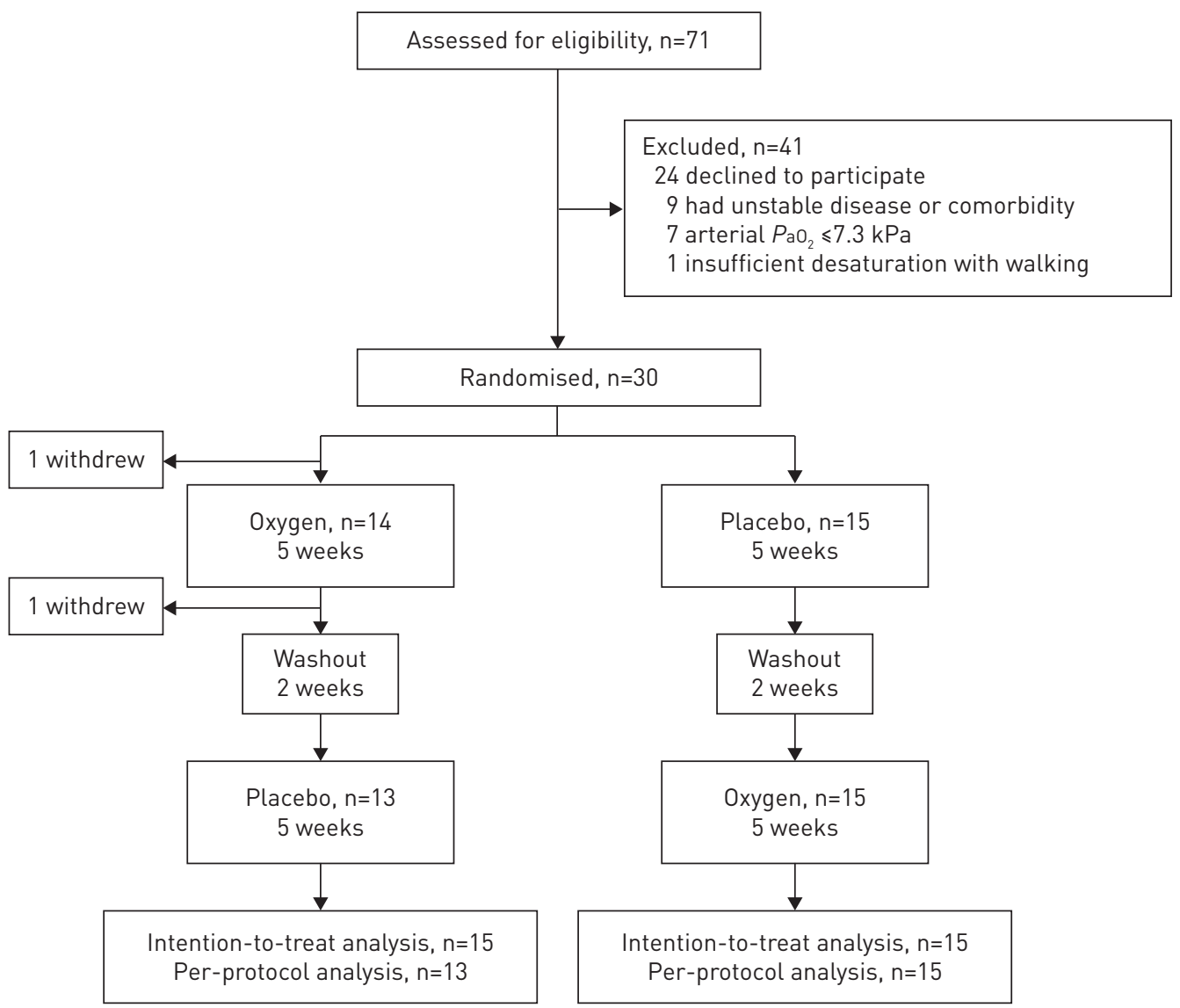

FIGURE 1 Patient flow chart. $\mathrm{PaO}_{2}$ : arterial oxygen tension.

of secondary outcomes was performed in the per-protocol population with all available data. For the NYHA class, treatment effects were estimated by random-effects ordered logistic regression and expressed as odds ratio $(95 \% \mathrm{CI})$. A probability of $\mathrm{p}<0.05$ was assumed as statistically significant.

\section{Results}

Patients

The patient flow chart is illustrated in figure 1. Patient characteristics are shown in table 1. 30 patients with $\mathrm{PAH} / \mathrm{CTEPH}$ were randomised and represented the intention-to-treat population. One patient withdrew consent after randomisation for personal reasons, another after completing the first treatment period due to newly detected breast cancer and pending treatment. For the intention-to-treat analysis, the missing data of these two patients were replaced by multiple imputation. The per-protocol analysis included 28 patients who completed the entire protocol.

\section{Main outcomes}

Table 2 presents the main outcomes. Over the course of the 5 weeks DOXT, the 6MWD increased by a mean $(95 \% \mathrm{CI})$ of $19(6-32) \mathrm{m}$, while the corresponding change with placebo was $-1(-11-13) \mathrm{m}$. The mean between-treatment difference (treatment effect) was $18(1-35) \mathrm{m}(\mathrm{p}=0.042)$ in favour of DOXT; the effect size was $0.40(0.01-0.79)$. The per-protocol analysis and the analysis with adjustment for baseline $6 \mathrm{MWD}$, age, sex and treatment order (figure 2, supplementary table S1) revealed consistent results. The heart rate at the end of the 6MWT increased to a higher value over the course of 5 weeks DOXT period compared to placebo and patients rated their dyspnoea at the end of the 6MWT slightly higher with DOXT than with placebo. The SF-36 physical functioning scale revealed an increase over the course of the 5 weeks DOXT, while there was no significant change with placebo, resulting in a between-treatment difference of $6(1-11)$ points $(p=0.029)$ in favour of DOXT; the effect size was $0.50(0.05-0.95)$. Results were similar in the intention-to-treat and the per-protocol analyses, and after adjustment for baseline scores, age, sex and treatment order (figure 2, supplementary table S2). The PH class (PAH and CTEPH) was not a significant predictor of the main outcomes (supplementary table S4). 


\begin{tabular}{|c|c|}
\hline Participants/female & $30 / 20$ \\
\hline Age years & $60 \pm 15$ \\
\hline \multicolumn{2}{|l|}{ Pulmonary hypertension classification } \\
\hline Pulmonary arterial hypertension & $14(47)$ \\
\hline Idiopathic & $12(40)$ \\
\hline Connective tissue disease-related & $2(7)$ \\
\hline CTEPH & $16(53)$ \\
\hline \multicolumn{2}{|c|}{ Right heart catheter data and systemic blood pressure } \\
\hline Mean pulmonary artery pressure $\mathrm{mmHg}$ & $39 \pm 11$ \\
\hline Pulmonary artery wedge pressure $\mathrm{mmHg}$ & $11 \pm 3$ \\
\hline Right atrial pressure $\mathrm{mmHg}$ & $8 \pm 4$ \\
\hline Cardiac index $\mathrm{L} \cdot \mathrm{min}^{-1} \cdot \mathrm{m}^{-2}$ & $3.0 \pm 0.8$ \\
\hline Pulmonary vascular resistance Wood units & $5.8 \pm 3.0$ \\
\hline Mixed venous oxygen saturation $\%$ & $67 \pm 8$ \\
\hline Heart rate beats $\cdot \mathrm{min}^{-1}$ & $70 \pm 12$ \\
\hline Systemic blood pressure, systolic $\mathrm{mmHg}$ & $126 \pm 18$ \\
\hline Systemic blood pressure, diastolic $\mathrm{mmHg}$ & $75 \pm 13$ \\
\hline \multicolumn{2}{|l|}{ Arterial blood gas analysis } \\
\hline $\mathrm{pH}$ & $7.42 \pm 0.04$ \\
\hline $\mathrm{PaO}_{2} \mathrm{kPa}$ & $10.0 \pm 1.7$ \\
\hline $\mathrm{PaCO}_{2} \mathrm{kPa}$ & $4.5 \pm 0.5$ \\
\hline \multicolumn{2}{|l|}{ Lung function } \\
\hline FEV $1 \%$ pred & $88 \pm 22$ \\
\hline FVC \% pred & $93 \pm 22$ \\
\hline FEV1/FVC \% & $77 \pm 8$ \\
\hline DLco \% pred & $69 \pm 15$ \\
\hline \multicolumn{2}{|l|}{ Pulmonary hypertension treatment } \\
\hline Endothelin receptor antagonist & $19(63)$ \\
\hline Phosphodiesterase- 5 inhibtor & $14(47)$ \\
\hline Soluble guanylate cyclase stimulator & $8(28)$ \\
\hline Prostanoids & $3(10)$ \\
\hline Combination therapy & $11(37)$ \\
\hline
\end{tabular}

On average, treatment adherence with DOXT was $13.2 \mathrm{~h} \cdot \mathrm{day}^{-1}$ which was less than the recommended use of $>16 \mathrm{~h} \cdot$ day $^{-1}$. Nevertheless, adherence with DOXT was significantly greater than that with placebo by a mean of $2.8 \mathrm{~h} \cdot \mathrm{day}^{-1}$ (95\% CI 0.3 to $\left.5.3, \mathrm{p}=0.026\right)$.

\section{Secondary outcomes}

Over the course of 5 weeks of DOXT, the proportion of patients in NYHA functional classes 1 and 2 increased, while the proportion of patients in classes 3 and 4 decreased (table 3). With placebo, there was an increase in the proportion of patients in classes 1 and 4 and a decrease in classes 2 and 3 . Correspondingly, logistic regression analysis indicated a beneficial effect of DOXT on the NYHA class as reflected in a low odds ratio for an increase by one class over the 5 weeks of DOXT of 0.13 (95\% CI 0.03 $0.61, \mathrm{p}=0.010$ ) compared to placebo (table 3 ). The SF-36 physical and mental component summary scores and the CAMPHOR quality of life symptoms and activity domains were similar at the end of the treatment periods with DOXT and placebo.

Echocardiography at the end of treatment periods did not reveal differences between DOXT and placebo in terms of the elevated TPG, the estimated right atrial pressure and the indices of right ventricular function (RVFAC, TAPSE) (table 3). However, there was a significant decrease in the right ventricular systolic and diastolic area at the end of the DOXT compared to the placebo period (table 3).

Arterial blood gas analyses while breathing ambient air did not show any significant changes in $\mathrm{PaO}_{2}$ and arterial carbon dioxide tension with treatment (table 3).

The sleep studies revealed mild hypoxaemia at baseline and an ODI and AHI within the normal range (table 3). At the end of the 5-week treatment periods (performed while using the corresponding treatment) the mean nocturnal $\mathrm{SpO}_{2}$ was increased with DOXT and unchanged with placebo. In addition, 


\begin{tabular}{|c|c|c|c|c|c|c|c|c|}
\hline & \multicolumn{3}{|c|}{ Placebo (ambient air) } & \multicolumn{3}{|c|}{ Domiciliary oxygen therapy } & \multicolumn{2}{|c|}{ Treatment effect $^{\#}$} \\
\hline & $\begin{array}{l}\text { Beginning of } \\
\text { treatment period }\end{array}$ & $\begin{array}{l}\text { End of 5-week } \\
\text { treatment period }\end{array}$ & $\begin{array}{l}\text { Mean change } \\
(95 \% \mathrm{CI})\end{array}$ & $\begin{array}{l}\text { Beginning of } \\
\text { treatment period }\end{array}$ & $\begin{array}{l}\text { End of 5-week } \\
\text { treatment period }\end{array}$ & $\begin{array}{c}\text { Mean change } \\
(95 \% \mathrm{Cl})\end{array}$ & $\begin{array}{l}\text { Mean difference of } \\
\text { change }(95 \% \mathrm{CI})\end{array}$ & p-value \\
\hline \multicolumn{9}{|l|}{ 6MWT (breathing ambient air) } \\
\hline \multicolumn{9}{|l|}{ 6MWD m } \\
\hline ITT & $484 \pm 113$ & $485 \pm 113$ & $1(-11-13)$ & $478 \pm 113$ & $497 \pm 114$ & $19(6-32)$ & $18(1-35)$ & 0.042 \\
\hline Per-protocol & $484 \pm 113$ & $484 \pm 107$ & $0(-12-11)$ & $477 \pm 107$ & $495 \pm 101$ & $18(6-30)$ & $19(2-35)$ & 0.028 \\
\hline Heart rate at rest L. $\min ^{-1}$ & $84 \pm 15$ & $79 \pm 14$ & $-5(-10-0)$ & $84 \pm 14$ & $76 \pm 13$ & $-8(-12--3)$ & $-3(-10-4)$ & 0.427 \\
\hline $\begin{array}{l}\text { Heart rate at end } \\
\quad \text { exercise } L \cdot \mathrm{min}^{-1}\end{array}$ & $122 \pm 19$ & $114 \pm 19$ & $-7(-13--1)$ & $114 \pm 19$ & $120 \pm 18$ & $6(-1-12)$ & $13(4-22)$ & 0.003 \\
\hline $\mathrm{SpO}_{2}$ at rest $\%$ & $95 \pm 3$ & $95 \pm 3$ & $0(-1-2)$ & $95 \pm 3$ & $94 \pm 3$ & $-1(-2-0)$ & $-1(-3-0)$ & 0.136 \\
\hline $\mathrm{SpO}_{2}$ at end exercise $\%$ & $88 \pm 5$ & $89 \pm 5$ & $1(-1-3)$ & $89 \pm 5$ & $88 \pm 5$ & $-1(-3-1)$ & $-2(-5-1)$ & 0.119 \\
\hline $\mathrm{SpO}_{2}$ desaturation with walk & $-7 \pm 4$ & $-7 \pm 5$ & $1(-1-3)$ & $-6 \pm 5$ & $-6 \pm 5$ & $0(-2-2)$ & $-1(-4-2)$ & 0.604 \\
\hline Dyspnoea Borg CR10 score & $5.2 \pm 2.7$ & $5.1 \pm 2.6$ & $-0.2(-1.0-0.7)$ & $4.3 \pm 2.6$ & $5.5 \pm 2.5$ & $1.1(0.2-2.0)$ & $1.3(0.1-2.5)$ & 0.036 \\
\hline \multicolumn{9}{|l|}{ Quality of life } \\
\hline \multicolumn{9}{|l|}{$\begin{array}{l}\text { SF-36 physical functioning } \\
\text { scale } \%\end{array}$} \\
\hline ITT & $54 \pm 29$ & $52 \pm 29$ & $-2(-6-2)$ & $52 \pm 29$ & $56 \pm 29$ & $4(0-8)$ & $6(1-11)$ & 0.029 \\
\hline Per-protocol & $56 \pm 27$ & $54 \pm 26$ & $-2(-6-2)$ & $53 \pm 26$ & $57 \pm 26$ & $4(0-8)$ & $6(1-12)$ & 0.022 \\
\hline \multicolumn{9}{|l|}{ Treatment adherence } \\
\hline $\begin{array}{l}\text { Mean concentrator } \\
\text { use } h \cdot \text { night }^{-1}\end{array}$ & & $10.4 \pm 6.7$ & & & $13.2 \pm 6.9$ & & $2.8(0.3-5.3)$ & 0.026 \\
\hline
\end{tabular}



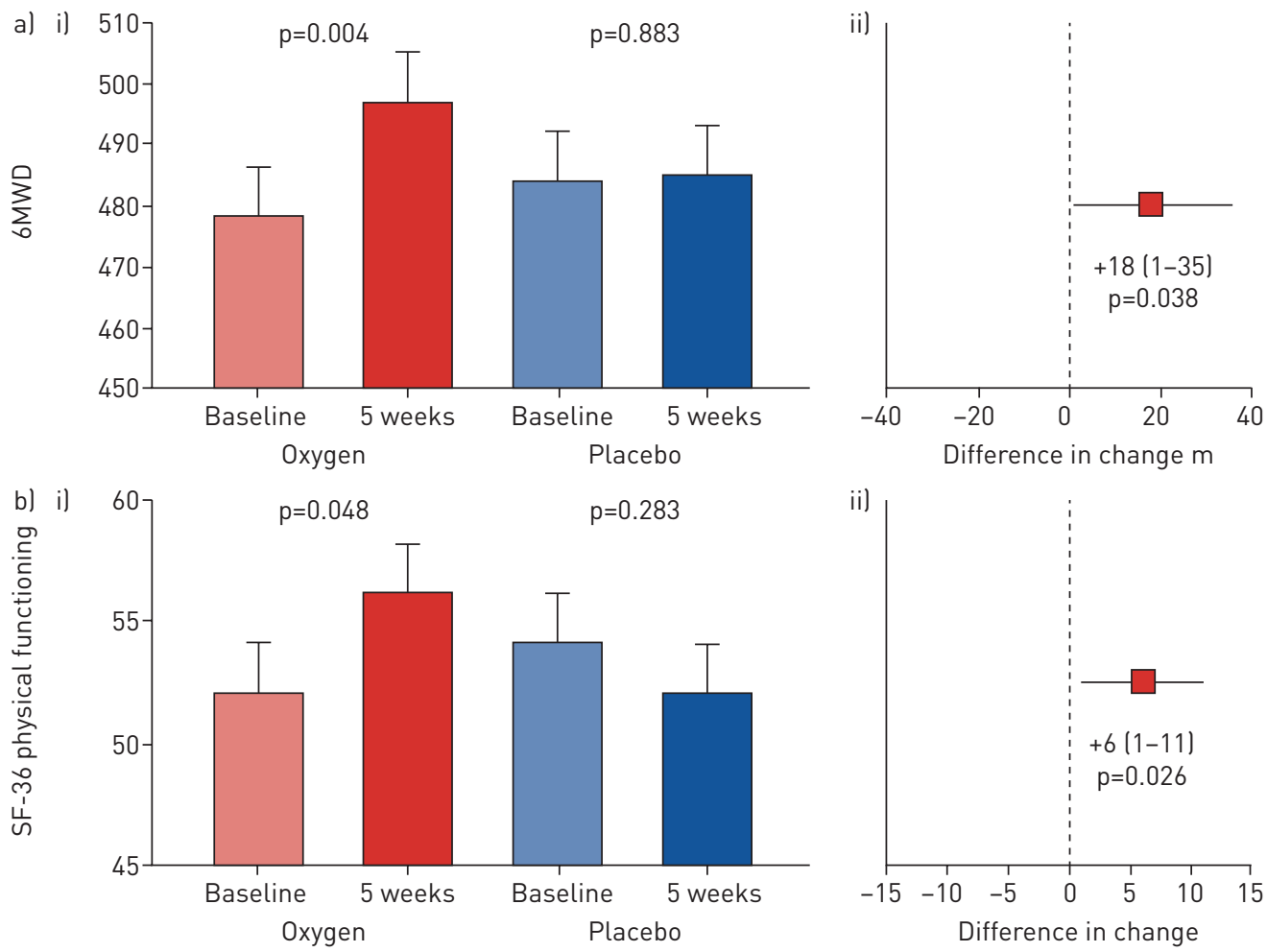

FIGURE 2 Effect of domiciliary oxygen therapy for 5 weeks on the a) 6-minute walk distance (6MWD) and b) physical functioning scale of the 36-item short form medical outcome quality-of-life questionnaire (SF-36). Data are presented as i) mean \pm SE adjusted values and ii) treatment effect of oxygen compared to placebo calculated as adjusted mean difference $(95 \% \mathrm{Cl}$ ) of changes during oxygen therapy minus corresponding vales during placebo therapy (regression models are shown in supplementary tables S1 and S2).

there was a slight decrease in the ODI with DOXT compared to placebo $(p=0.011)$ and a trend for a decrease in mean nocturnal heart rate with DOXT ( $\mathrm{p}=0.058)$.

Cognitive testing revealed an increased speed in Stroop 1 assessed at the end of the DOXT compared to the placebo period (table 3).

Activity recordings over the course of week 5 of the treatment periods did not show any difference in the estimated number of steps per day (table 3 ).

No serious adverse events occurred. Episodes of mild epistaxis were reported by seven out of 30 patients during the DOXT period and by six out of 30 patients during the placebo period.

\section{Discussion}

The current randomised placebo-controlled double-blind, crossover trial is the first to evaluate the effect of DOXT in patients suffering from PAH/CTEPH with mild resting hypoxaemia and oxygen desaturation during exercise. The results demonstrate that 5 weeks of DOXT during nights and rest at home leads to a significant increase in the 6MWD and in the SF-36 physical functioning quality-of-life score compared to placebo treatment. Moreover, DOXT improved NYHA functional class and aspects of cognitive performance and it increased and stabilised the nocturnal $\mathrm{SpO}_{2}$. The current trial provides important new evidence supporting the use of DOXT as a valuable adjunct to medical therapy in selected patients with PAH/ CTEPH. It corroborates and extends our two previous randomised placebo-controlled trials in patients with $\mathrm{PAH} / \mathrm{CTEPH}$ demonstrating an improvement in cycling endurance with oxygen administration and in the $6 \mathrm{MWD}$ after 1 week of nocturnal oxygen therapy in those with sleep disordered breathing $[4,9,22]$.

Until recently, there has been a lack of evidence supporting a benefit of DOXT in patients with $\mathrm{PH}$. Acknowledging this limitation, the authors of the 2015 European Society of Cardiology/European Respiratory Society guidelines [1] have suggested the use of data from studies in patients with COPD performed $>30$ years ago $[11,23,24]$ as a guidance to prescribe DOXT to patients with $\mathrm{PH}$ "when $\mathrm{PaO}_{2}$ is consistently $<8.0 \mathrm{kPa}$ " and to consider ambulatory oxygen "when there is evidence of symptomatic benefit and correctable desaturation during exercise". Considering the fundamentally different pathophysiology of 
TABLE 3 Secondary outcomes

\section{Baseline}

End of 5-week

placebo treatment

(ambient air)

\section{End of 5-week \\ domiciliary \\ oxygen therapy}

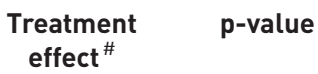

Treatment p-value effect $^{\#}$

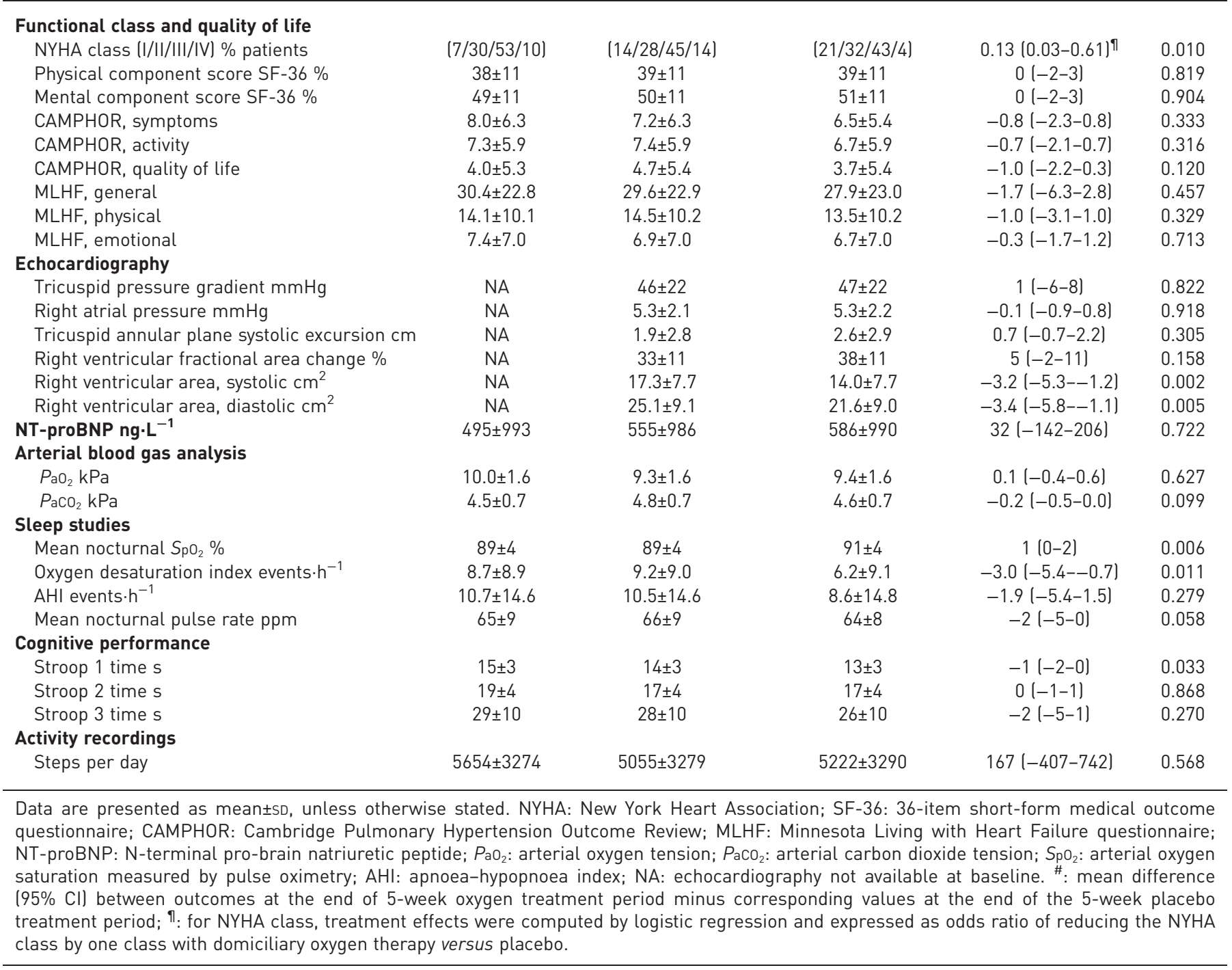

COPD and PAH/CTEPH, the urgent need for robust evidence evaluating the role of oxygen therapy in $\mathrm{PAH} / \mathrm{CTEPH}$ is obvious. The current randomised placebo-controlled trial address this gap in knowledge by demonstrating a beneficial effect of DOXT in a specific setting, i.e. in patients with $\mathrm{PAH} / \mathrm{CTEPH}$ who have mild hypoxaemia at rest that exacerbates during physical activity.

The symptoms and limitations of exercise performance in $\mathrm{PH}$ and its distinct characteristic, exercise-induced hypoxaemia, can be attributed to several pathophysiological mechanisms: impaired pulmonary gas exchange due to ventilation-perfusion mismatch $[4,7]$, limitation of pulmonary vasculature recruitment, excessive rise in pulmonary vascular resistance (PVR) hindering an adequate increase in cardiac output by the already stressed right ventricle [25], increased respiratory drive leading to hypocapnia with inefficient ventilation and worsening of PVR due to hypoxic pulmonary vasoconstriction with progressive arterial and mixed-venous hypoxaemia during exercise.

In healthy individuals [26] and patients with PAH/CTEPH [4], we showed that breathing oxygen-enriched air during cycling exercise substantially increased performance by acting on the cited pathophysiological mechanisms. In particular, breathing oxygen-enriched air reduced the ventilatory response to exercise that is typically excessive in $\mathrm{PH}$, thereby reduced the ventilatory equivalents for carbon dioxide output and improved the arterial oxygenation along with a greater availability of oxygen in muscles and in the 
brain $[4,26]$. In the current study, even though oxygen therapy was not provided during assessment of the $6 \mathrm{MWD}$ at the end of the 5 weeks of DOXT, performance was improved compared to placebo. Presumably, alleviation of hypoxaemia by DOXT reflected in the higher nocturnal $\mathrm{SpO}_{2}$ with DOXT (table 3) may have contributed to a reduction of the ventilatory drive with enhanced ventilatory efficiency and a stabilising effect on ventilation, as suggested by the reduced ODI (table 3). Moreover, the trend for a reduction in nocturnal pulse rate during DOXT is consistent with a reduction in sympathetic tone. Together, these effects of DOXT may have promoted a reduction in the PVR that was sustained even during temporary discontinuation of oxygen administration during daytime and the 6MWD tests. The reduction in the right ventricular dimensions documented by echocardiography (table 3) is consistent with right ventricular unloading by DOXT.

Physical activity is of paramount importance for living a normal life, and inability to participate in daily physical activities is associated with a reduced quality of life [27]. Thus, the improvements in the NYHA functional class and in the physical functioning scale of SF-36 quality of life by DOXT are important, patient-centred outcomes of our study (table 3). The quality-of-life domains assessed with the PH-specific CAMPHOR questionnaire did not significantly change during the study. Consistent with the current results, our earlier trial in patients with $\mathrm{PAH} / \mathrm{CTEPH}$ and sleep-related breathing disturbances revealed improvements in the 6MWD after 1 week of nocturnal oxygen therapy in association with an increase in the SF-36 physical functioning score. However, physical activity assessed during the last week of DOXT or placebo by an accelerometer showed no difference between the two treatment periods. Apparently, the daily activity was not adapted to the greater performance ability with DOXT or actimetry was not sensitive enough to capture subtle changes.

We have shown previously that cerebral tissue oxygenation is correlated to cognitive performance in $\mathrm{PAH} /$ CTEPH patients [8], and that a lower cerebral tissue oxygenation during exercise is prevented with oxygen therapy [4, 18]. Thus, by improving systemic and possibly cerebral oxygenation DOXT may have enhanced cerebral functions (table 3).

DOXT was well tolerated by the patients and, apart from occasional minor nasal bleeding, did not have any relevant undesirable effects. Nevertheless, the inconvenience of wearing a nasal cannula during the night and daytime may have prevented a positive effect of DOXT in certain quality-of-life domains other than physical functioning. Interestingly, patients used DOXT for more hours per day $\left(+2.8 \mathrm{~h} \cdot \mathrm{day}^{-1}\right.$ on average; table 1) than placebo, raising the possibility that the lack of a perceived beneficial effect of placebo might have negatively influenced adherence.

Although oxygen therapy was applied in the current trial over a much longer time period (5 weeks) than in previous randomised studies (up to 1 week) [9] we cannot exclude that an even more prolonged treatment over several months or years and a greater adherence to DOXT than the observed mean use of $13.2 \mathrm{~h} \cdot \mathrm{day}^{-1}$ would have improved pulmonary haemodynamics and important outcomes such as time to clinical worsening. Therefore, the current results may serve as a valuable basis for designing larger trials evaluating long-term DOXT in patients with PH. The effect size achieved with DOXT was small to moderate, but still considerable, taking into account that patients were already on $\mathrm{PH}$-targeted drugs. Moreover, we cannot exclude that the estimated treatment effect of DOXT was diluted to some degree by the crossover design of our trial even though our analysis did not indicate any carry-over or order effect (supplementary tables S1 and S2).

In conclusion, our randomised, placebo-controlled trial demonstrates that 5 weeks of DOXT improves exercise performance, quality of life and NYHA functional class in patients with PAH/CTEPH who have exercise-induced hypoxaemia. Therefore, in addition to its beneficial effects in PAH/CTEPH with sleep-related breathing disorders [9], DOXT has the potential to serve as a valuable adjunct to PH-targeted drug treatment in patients with PAH/CTEPH who show mild hypoxaemia at rest that is exacerbated during exercise.

Author contributions: S. Ulrich and K.E. Bloch contributed to the conception and design. S. Saxer, E.D. Hasler, E.I. Schwarz, S.R. Schneider, M. Furian, P.R. Bader, M. Lichtblau, K.E. Bloch and S. Ulrich contributed to acquisition, analysis, or interpretation of data. S. Ulrich and S. Saxer drafted the manuscript. All authors revised the manuscript critically for important intellectual content. S. Ulrich is the guarantor of the study.

Conflict of interest: S. Ulrich reports grants from Zurich Lung League and Swiss National Science Foundation, during the conduct of the study; grants and personal fees from Actelion SA and Orpha Swiss, personal fees from Bayer SA and MSD, outside the submitted work. S. Saxer has nothing to disclose. E.D. Hasler has nothing to disclose. E.I. Schwarz has nothing to disclose. S.R. Schneider has nothing to disclose. M. Furian has nothing to disclose. P.R. Bader has nothing to disclose. M. Lichtblau has nothing to disclose. K.E. Bloch reports grants from Zurich Lung League and Swiss National Science Foundation, during the conduct of the study. 
Support statement: This work was supported by grants from the Swiss National Science Foundation (grant number 146266) and the Lunge Zurich, Switzerland. Funding information for this article has been deposited with the Crossref Funder Registry.

\section{References}

1 Galiè N, Humbert M, Vachiery JL, et al. 2015 ESC/ERS Guidelines for the diagnosis and treatment of pulmonary hypertension: The Joint Task Force for the Diagnosis and Treatment of Pulmonary Hypertension of the European Society of Cardiology (ESC) and the European Respiratory Society (ERS). Eur Heart J 2016; 37: 67-119.

2 Cenedese E, Speich R, Dorschner L, et al. Measurement of quality of life in pulmonary hypertension and its significance. Eur Respir J 2006; 28: 808-815.

3 Cima K, Twiss J, Speich R, et al. The German adaptation of the Cambridge Pulmonary Hypertension Outcome Review (CAMPHOR). Health Qual Life Outcomes 2012; 10: 110.

4 Ulrich S, Hasler ED, Saxer S, et al. Effect of breathing oxygen-enriched air on exercise performance in patients with precapillary pulmonary hypertension: randomized, sham-controlled cross-over trial. Eur Heart J 2017; 38: $1159-1168$

5 Marshall C, Marshall B. Site and sensitivity for stimulation of hypoxic pulmonary vasoconstriction. J Appl Physiol Environ Exerc Physiol 1983; 55: 711-716.

$6 \quad$ Naeije R, Chesler N. Pulmonary circulation at exercise. Compr Physiol 2012; 2: 711-741.

7 Sun XG, Hansen JE, Oudiz RJ, et al. Exercise pathophysiology in patients with primary pulmonary hypertension. Circulation 2001; 104: 429-435.

8 Müller-Mottet S, Hildenbrand FF, Keusch S, et al. Effects of exercise and vasodilators on cerebral tissue oxygenation in pulmonary hypertension. Lung 2015; 193: 113-120.

9 Ulrich S, Keusch S, Hildenbrand FF, et al. Effect of nocturnal oxygen and acetazolamide on exercise performance in patients with pre-capillary pulmonary hypertension and sleep-disturbed breathing: randomized, double-blind, cross-over trial. Eur Heart I 2015; 36: 615-623.

10 Muller-Mottet S, Hasler E, Opitz I, et al. Chronic thromboembolic pulmonary hypertension. Cardiovasc Med 2014; 17: 328-333.

11 Weitzenblum E, Sautegeau A, Ehrhart M, et al. Long-term oxygen therapy can reverse the progression of pulmonary hypertension in patients with chronic obstructive pulmonary disease. Am Rev Respir Dis 1985; 131: 493-498.

12 Ulrich S, Schneider SR, Bloch KE. Effect of hypoxia and hyperoxia on exercise performance in healthy individuals and in patients with pulmonary hypertension: a systematic review. J Appl Physiol 2017; 123: 1657-1670.

13 Pepke-Zaba J, Gilbert C, Collings L, et al. Sildenafil improves health-related quality of life in patients with pulmonary arterial hypertension. Chest 2008; 133: 183-189.

14 Schulz R, Baseler G, Ghofrani HA, et al. Nocturnal periodic breathing in primary pulmonary hypertension. Eur Respir J 2002; 19: 658-663.

15 Ware JE, Snow KK, Kosinski M, et al. SF-36 Health Survey: Manual and Interpretation Guide. Boston, The Medical Outcome Trust, New England Medical Center, 1993.

16 Rudski LG, Lai WW, Afilalo J, et al. Guidelines for the echocardiographic assessment of the right heart in adults: a report from the American Society of Echocardiography endorsed by the European Association of Echocardiography, a registered branch of the European Society of Cardiology, and the Canadian Society of Echocardiography. J Am Soc Echocardiogr 2010; 23: 685-713.

17 Ulrich S, Fischler M, Speich R, et al. Sleep-related breathing disorders in patients with pulmonary hypertension. Chest 2008; 133: 1375-1380

18 Somaini G, Stamm A, Müller-Mottet S, et al. Disease-targeted treatment improves cognitive function in patients with precapillary pulmonary hypertension. Respiration 2015; 90: 376-383.

19 Hill K, Dolmage TE, Woon L, et al. Measurement properties of the SenseWear armband in adults with chronic obstructive pulmonary disease. Thorax 2010; 65: 486-491.

20 White IR, Royston P, Wood AM. Multiple imputation using chained equations: issues and guidance for practice. Stat Med 2011; 30: 377-399.

21 Kazis LE, Anderson JJ, Meenan RF. Effect sizes for interpreting changes in health status. Med Care 1989; 27: Suppl. 3, S178-S189.

22 Schumacher DS, Müller-Mottet S, Hasler ED, et al. Effect of oxygen and acetazolamide on nocturnal cardiac conduction, repolarization, and arrhythmias in precapillary pulmonary hypertension and sleep-disturbed breathing. Chest 2014; 146: 1226-1236.

23 Nocturnal Oxygen Therapy Trial Group. Continuous or nocturnal oxygen therapy in hypoxemic chronic obstructive lung disease: a clinical trial. Ann Intern Med 1980; 93: 391-398.

24 Long term domiciliary oxygen therapy in chronic hypoxic cor pulmonale complicating chronic bronchitis and emphysema. Report of the Medical Research Council Working Party. Lancet 1981; 1: 681-686.

25 Hasler ED, Müller-Mottet S, Furian M, et al. Pressure-flow during exercise catheterization predicts survival in pulmonary hypertension. Chest 2016; 150: 57-67.

26 Ulrich S, Hasler ED, Müller-Mottet S, et al. Mechanisms of improved exercise performance under hyperoxia. Respiration 2017; 93: 90-98.

27 Heesch KC, van Uffelen JG, van Gellecum YR, et al. Dose-response relationships between physical activity, walking and health-related quality of life in mid-age and older women. J Epidemiol Community Health 2012; 66 : 670-677. 